Supporting Information for

\title{
Density Functional Theory Study of Uranium(VI) Aquo Chloro Complexes in Aqueous Solution.
}

Michael Bühl,* Nicolas Sieffert, Volodymyr Golubnichiy, and Georges Wipff

Max-Planck-Institut für Kohlenforschung, Kaiser-Wilhelm-Platz 1, D-45470 Mülheim an der Ruhr, Germany, and UMR 7177 CNRS, Laboratoire MSM, Institut de Chimie, 4 rue Blaise Pascal, 67000 Strasbourg, France, E-mail: buehl@mpi-muelheim.mpg.de

Table S1: Geometrical parameters (bond distances in $\AA)^{a}$ of complexes $\mathbf{1}$ - 5, computed with the LANL ECP and valence basis on $\mathrm{U}[6-31 \mathrm{G}(\mathrm{d}, \mathrm{p})$ basis elsewhere].

\begin{tabular}{|c|c|c|c|c|c|c|c|}
\hline \multicolumn{2}{|c|}{ Comp. bond } & \multirow{2}{*}{$\begin{array}{r}\text { BLYP } \\
1.82\end{array}$} & \multirow{2}{*}{$\begin{array}{r}\text { PCM/ } \\
\text { BLYP } \\
1.82\end{array}$} & \multirow{2}{*}{$\begin{array}{r}\text { BP86 } \\
1.80\end{array}$} & \multirow{2}{*}{$\begin{array}{r}\mathrm{PCM} / \\
\mathrm{BP} 86\end{array}$} & \multirow{2}{*}{$\begin{array}{r}\text { B3LYP } \\
1.77\end{array}$} & \multirow{2}{*}{$\begin{array}{r}\begin{array}{r}\text { PCM/ } \\
\text { B3LYP }\end{array} \\
1.78\end{array}$} \\
\hline $1 a$ & $r(\mathrm{U}=\mathrm{O})$ & & & & & & \\
\hline & $r(\mathrm{U}-\mathrm{Cl})$ & 2.66 & 2.74 & 2.63 & 2.71 & 2.62 & 2.73 \\
\hline & $r(\mathrm{U}-\mathrm{O})$ & 2.62 & 2.52 & 2.60 & 2.50 & 2.56 & 2.49 \\
\hline & $r\left(\mathrm{U}^{-\mathrm{O}^{\prime}}\right)$ & 2.56 & 2.51 & 2.54 & 2.50 & 2.57 & 2.51 \\
\hline \multirow[t]{4}{*}{$1 b$} & $r(\mathrm{U}=\mathrm{O})$ & 1.81 & 1.82 & 1.80 & 1.80 & 1.77 & 1.78 \\
\hline & $r(\mathrm{U}-\mathrm{Cl})$ & 2.60 & 2.68 & 2.58 & 2.66 & 2.57 & 2.68 \\
\hline & $r(\mathrm{U}-\mathrm{O})$ & 2.51 & 2.44 & 2.49 & 2.42 & 2.49 & 2.41 \\
\hline & $r\left(\mathrm{U}-\mathrm{O}^{\prime}\right)$ & 2.53 & 2.44 & 2.50 & 2.41 & 2.51 & 2.42 \\
\hline \multirow[t]{4}{*}{$c i s-\mathbf{2 a}$} & $r(\mathrm{U}=\mathrm{O})$ & 1.82 & 1.82 & 1.81 & 1.81 & 1.78 & 1.78 \\
\hline & $r(\mathrm{U}-\mathrm{Cl})$ & 2.71 & 2.76 & 2.69 & 2.71 & 2.69 & 2.75 \\
\hline & $r(\mathrm{U}-\mathrm{O})$ & 2.69 & 2.57 & 2.66 & 2.54 & 2.65 & 2.53 \\
\hline & $r\left(\mathrm{U}^{\prime} \mathrm{O}^{\prime}\right)$ & 2.67 & 2.60 & 2.63 & 2.57 & 2.62 & 2.55 \\
\hline \multirow[t]{3}{*}{ cis-2b } & $r(\mathrm{U}=\mathrm{O})$ & 1.82 & 1.82 & 1.81 & 1.81 & 1.78 & 1.78 \\
\hline & $r(\mathrm{U}-\mathrm{Cl})$ & 2.67 & 2.72 & 2.64 & 2.69 & 2.64 & 2.70 \\
\hline & $r(\mathrm{U}-\mathrm{O})$ & 2.58 & 2.46 & 2.56 & 2.44 & 2.56 & 2.43 \\
\hline \multirow[t]{4}{*}{$t r .-\mathbf{2 a}$} & $r(\mathrm{U}=\mathrm{O})$ & 1.82 & 1.82 & 1.81 & 1.81 & 1.78 & 1.78 \\
\hline & $r(\mathrm{U}-\mathrm{Cl})$ & 2.75 & 2.78 & 2.73 & 2.75 & 2.73 & 2.77 \\
\hline & $r(\mathrm{U}-\mathrm{O})$ & 2.58 & 2.51 & 2.56 & 2.51 & 2.54 & 2.52 \\
\hline & $r\left(\mathrm{U}-\mathrm{O}^{\prime}\right)$ & 2.63 & 2.58 & 2.60 & 2.54 & 2.60 & 2.52 \\
\hline \multirow[t]{3}{*}{$t r .-2 \mathbf{b}$} & $r(\mathrm{U}=\mathrm{O})$ & 1.82 & 1.82 & 1.81 & 1.81 & 1.78 & 1.78 \\
\hline & $r(\mathrm{U}-\mathrm{Cl})$ & 2.68 & 2.72 & 2.66 & 2.69 & 2.66 & 2.71 \\
\hline & $r(\mathrm{U}-\mathrm{O})$ & 2.57 & 2.45 & 2.55 & 2.43 & 2.54 & 2.42 \\
\hline
\end{tabular}




\begin{tabular}{|c|c|c|c|c|c|c|c|}
\hline \multirow[t]{4}{*}{$\operatorname{cis} \mathbf{- 3 a}$} & $r(\mathrm{U}=\mathrm{O})$ & 1.83 & 1.83 & 1.81 & 1.82 & 1.78 & 1.78 \\
\hline & $r(\mathrm{U}-\mathrm{Cl})$ & 2.70 & 2.78 & 2.66 & 2.74 & 2.67 & 2.77 \\
\hline & $r\left(\mathrm{U}-\mathrm{Cl}^{\prime}\right)$ & 2.86 & 2.82 & 2.82 & 2.78 & 2.83 & 2.80 \\
\hline & $r(\mathrm{U}-\mathrm{O})$ & 2.74 & 2.62 & 2.71 & 2.60 & 2.71 & 2.58 \\
\hline \multirow[t]{4}{*}{$t r .-\mathbf{3 a}$} & \multicolumn{2}{|c|}{$r(\mathrm{U}=\mathrm{O}) \rightarrow c i s-2 \mathbf{b} \cdot \mathrm{Cl}^{-}$} & \multicolumn{2}{|c|}{$1.83 \rightarrow c i s-2 \mathbf{b} \cdot \mathrm{Cl}^{-}$} & \multicolumn{2}{|c|}{$1.81 \rightarrow c i s-\mathbf{2 b} \cdot \mathrm{Cl}^{-}$} & 1.78 \\
\hline & $r(\mathrm{U}-\mathrm{Cl})$ & " & 2.84 & $"$ & 2.81 & " & 2.83 \\
\hline & $r\left(\mathrm{U}-\mathrm{Cl}^{\prime}\right)$ & " & 2.81 & $"$ & 2.77 & $"$ & 2.79 \\
\hline & $r(\mathrm{U}-\mathrm{O})$ & " & 2.59 & $"$ & 2.57 & $"$ & 2.55 \\
\hline \multirow[t]{4}{*}{$3 b$} & $r(\mathrm{U}=\mathrm{O})$ & 1.82 & 1.83 & 1.81 & 1.81 & 1.78 & 1.78 \\
\hline & $r(\mathrm{U}-\mathrm{Cl})$ & 2.70 & 2.75 & 2.67 & 2.71 & 2.68 & 2.73 \\
\hline & $r\left(\mathrm{U}-\mathrm{Cl}^{\prime}\right)$ & 2.78 & 2.75 & 2.75 & 2.72 & 2.75 & 2.73 \\
\hline & $r(\mathrm{U}-\mathrm{O})$ & 2.66 & 2.47 & 2.63 & 2.45 & 2.62 & 2.44 \\
\hline \multirow[t]{2}{*}{4} & $r(\mathrm{U}=\mathrm{O})$ & 1.83 & 1.83 & 1.82 & 1.82 & 1.78 & 1.78 \\
\hline & $r(\mathrm{U}-\mathrm{Cl})$ & 2.82 & 2.77 & 2.78 & 2.74 & 2.79 & 2.75 \\
\hline \multirow[t]{2}{*}{5} & $r(\mathrm{U}=\mathrm{O})$ & 1.79 & 1.81 & 1.80 & 1.80 & 1.76 & 1.77 \\
\hline & $r(\mathrm{U}-\mathrm{O})$ & 2.54 & 2.50 & 2.49 & 2.45 & 2.47 & 2.46 \\
\hline
\end{tabular}


Table S2: Computed energies and free energies (in $\mathrm{kcal} / \mathrm{mol}$ ) for the cumulated chloride binding energies of uranyl hydrate according to eq. 4 employing LANL-optimized geometries.

\begin{tabular}{|c|c|c|c|c|c|c|c|c|c|c|c|c|}
\hline \multirow{3}{*}{$\begin{array}{l}\text { level } \\
n \text { (cis/tr.) }\end{array}$} & \multicolumn{4}{|c|}{ BLYP } & \multicolumn{4}{|c|}{ BP86 } & \multicolumn{4}{|c|}{ B3LYP } \\
\hline & $\Delta E($ gas $) \triangle$ & $E / \mathrm{PCM}$ & $\Delta G / \mathrm{PCM}$ & $1 \quad \Delta E / \mathrm{PCM}^{a}$ & $\Delta E($ gas $)$ & $\Delta E / \mathrm{PCM}$ & $\Delta G / \mathrm{PCM}$ & $\Delta E / \mathrm{PCM}^{a}$ & $\Delta E($ gas $)$ & $\Delta E / \mathrm{PCM}$ & $\Delta G / \mathrm{PCM}$ & $\Delta E / \mathrm{PCM}^{a}$ \\
\hline & LANL & LANL & LANL & $\operatorname{SDD}(+)\left[\Delta E^{\mathrm{CP}}\right]$ & LANL & LANL & LANL & $\operatorname{SDD}(+)$ & LANL & LANL & LANL & $\operatorname{SDD}(+)\left[\Delta E^{\mathrm{CP}}\right]$ \\
\hline 1 & -203.8 & -5.8 & -5.4 & $-8.5[-9.5]$ & -202.2 & -6.7 & -9.2 & -9.2 & -196.8 & -2.6 & -4.2 & $-6.0[-7.0]$ \\
\hline 2 (cis) & -322.2 & -7.1 & -10.0 & $-13.2[-15.1]$ & -321.9 & -10.4 & -14.0 & -15.9 & -312.5 & -2.9 & -5.9 & $-9.3[-11.4]$ \\
\hline 2 (trans) & -324.8 & -7.0 & -11.1 & $-13.4[-15.3]$ & -324.7 & -9.6 & -12.9 & -15.3 & -317.0 & -3.3 & -5.9 & $-9.8[-11.9]$ \\
\hline 3 (cis) & -362.6 & -5.1 & -10.8 & $-14.9[-17.7]$ & -363.2 & -9.3 & -16.1 & -18.2 & -351.2 & 0.0 & -5.4 & $-9.7[-12.9]$ \\
\hline 3 (trans) & $-367.7^{b}$ & -5.2 & -11.3 & $-15.4[-18.3]$ & $-367.5^{b}$ & -9.3 & -15.3 & -18.8 & $-356.5^{b}$ & -0.5 & -5.7 & $-10.7[-13.9]$ \\
\hline 4 & $(-327.3)^{c}$ & +1.0 & -7.6 & $-12.6[-16.4]$ & $(-327.4)^{c}$ & -1.7 & -10.8 & -19.4 & $(-317.5)^{c}$ & +6.8 & -2.8 & $-6.9[-11.1]$ \\
\hline
\end{tabular}

${ }^{a}$ In square brackets: Counterpoise-corrected energies (see paper). ${ }^{b}$ Trans-3a optimized to cis-2b $\cdot \mathrm{Cl}^{-} \quad$ in the gas phase. ${ }^{c} \mathrm{Because}$ no $\left[\mathrm{UO}_{2}\left(\mathrm{H}_{2} \mathrm{O}\right) \mathrm{Cl}_{4}\right]^{2-}$ minimum can be located in the gas phase, these values in parentheses refer to a microsolvated $\left[\mathrm{UO}_{2} \mathrm{Cl}_{4}\right]^{2-}$. $\left(\mathrm{H}_{2} \mathrm{O}\right)$ complex; all other entries in this row are for the five-coordinate $\left[\mathrm{UO}_{2}\left(\mathrm{H}_{2} \mathrm{O}\right) \mathrm{Cl}_{4}\right]^{2-}$ species that can be optimized in the continuum. 
Table S3: Computed energies and free energies (in kcal/mol) for the cumulated chloride binding energies of uranyl hydrate according to eq. 4 employing LANL-optimized geometries.

\begin{tabular}{|c|c|c|c|c|c|c|c|c|c|c|c|c|}
\hline \multirow{3}{*}{$\begin{array}{l}\text { level } \\
n \text { (cis/tr.) }\end{array}$} & \multicolumn{4}{|c|}{ BLYP } & \multicolumn{4}{|c|}{ BP86 } & \multicolumn{4}{|c|}{ B3LYP } \\
\hline & $\Delta E($ gas $)$ & $E / \mathrm{PCM}$ & $\Delta G / \mathrm{PCM}$ & $1 \quad \Delta E / \mathrm{PCM}^{a}$ & $\Delta E($ gas $)$ & $\Delta E / \mathrm{PCM}$ & $\Delta G / \mathrm{PCM}$ & $\Delta E / \mathrm{PCM}^{a}$ & $\Delta E($ gas $)$ & $\Delta E / \mathrm{PCM}$ & $\Delta G / \mathrm{PCM}$ & $\Delta E / \mathrm{PCM}^{a}$ \\
\hline & ) LANL & LANL & LANL & $\mathrm{SDD}(+)\left[\Delta E^{\mathrm{CP}}\right]$ & LANL & LANL & LANL & $\mathrm{SDD}(+)$ & LANL & LANL & LANL & $\operatorname{SDD}(+)\left[\Delta E^{\mathrm{CP}}\right]$ \\
\hline 0 & 26.9 & 17.3 & 8.3 & $11.6[9.8]$ & 28.5 & 15.2 & 2.1 & 12.6 & 29.1 & 18.5 & 11.6 & 14.1 [12.2] \\
\hline 1 & 19.5 & 13.9 & 7.8 & $6.2[4.4]$ & 19.7 & 12.6 & 0.2 & 6.8 & 21.5 & 15.5 & 7.9 & 8.8 [6.9] \\
\hline 2 (cis) & 17.3 & 10.9 & 2.7 & $3.0[1.2]$ & 17.7 & 11.0 & -0.9 & 4.7 & 18.4 & 12.5 & 4.6 & 5.6 [3.7] \\
\hline $2($ trans $)$ & 19.0 & 10.8 & 4.1 & $3.7[1.8]$ & 19.7 & 10.5 & -2.2 & 4.7 & 21.6 & 12.9 & 3.6 & 6.5 [4.6] \\
\hline 3 (cis) & 12.0 & 7.6 & -0.4 & $0.0[-1.8]$ & 12.5 & 7.8 & -3.3 & 1.4 & 11.2 & 9.0 & 1.4 & $2.3[0.5]$ \\
\hline 3 (trans) & $17.1^{b}$ & 7.7 & 0.2 & $-0.5[-2.4]$ & 16.8 & 7.8 & -4.1 & 2.0 & $16.5^{b}$ & 9.6 & 1.6 & $3.4[-1.5]$ \\
\hline 4 & $(16.2)^{c}$ & 2.5 & -4.7 & $-4.9[-6.7]$ & & & & & $(16.7)^{c}$ & 3.6 & -2.1 & $-2.5[-4.4]$ \\
\hline
\end{tabular}

${ }^{a}$ In square brackets: Counterpoise-corrected energies (see paper). ${ }^{b}$ Trans-3a optimized to cis-2 $\mathbf{2} \cdot \mathrm{Cl}^{-} \quad$ in the gas phase. ${ }^{c} \mathrm{Because}$ no $\left[\mathrm{UO} \mathrm{O}_{2}\left(\mathrm{H}_{2} \mathrm{O}\right) \mathrm{Cl}_{4}\right]^{2-}$ minimum can be located in the gas phase, these values in parentheses refer to a microsolvated $\left[\mathrm{UO}_{2} \mathrm{Cl}_{4}\right]^{2-} \cdot\left(\mathrm{H}_{2} \mathrm{O}\right)$ complex; all other entries in this row are for the five-coordinate $\left[\mathrm{UO}_{2}\left(\mathrm{H}_{2} \mathrm{O}\right) \mathrm{Cl}_{4}\right]^{2-}$ species that can be optimized in the continuum. 


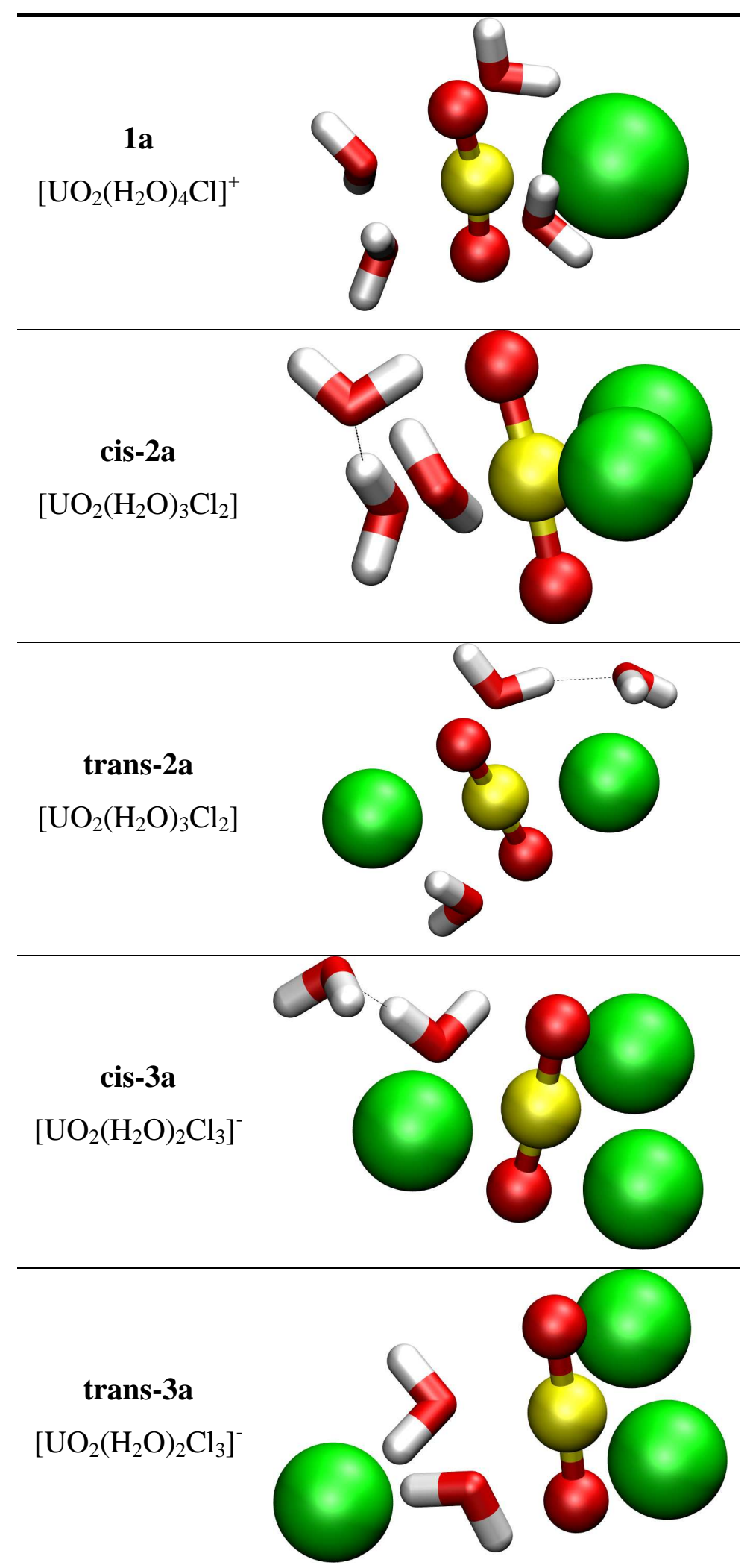

Figure S1 : Final snapshots of $\left[\mathrm{UO}_{2}\left(\mathrm{H}_{2} \mathrm{O}\right)_{5-n} \mathrm{Cl}_{n}\right]^{5-n}$ complexes $(\mathrm{n}=1 . .3)$, obtained after $12 \mathrm{ps}$ of CPMD/BLYP simulations in the gas phase. 
Cartesian coordinates of complexes optimized at the BLYP/LANL level in the gas phase (xyz format in $\AA$ ). In those cases, where a different conformation was located in the continuum, the corresponding PCm-optimized coordinates are given as well (denoted PCM).

16

Complex 1a

O 0.187124

U $\quad 0.227492$

O -0.383201

O 0.599934

Cl -2.321714

$-0.104004$

$-1.654956$

$-0.129521$

2.450307

$-0.347523$

0.293244

0.350475

O 2.346265

$-1.472215$

O $\quad-0.769839$

$-2.460712$

1.645234

1.916334

0.792606

O 2.099475

$\mathrm{H} \quad 2.920820$

$-1.888200$

$-0.236680$

0.000126

0.393565

H 2.746089

$-1.644580$

0.434080

H -1.371396

$-2.727323$

$-1.110545$

H -1.217343

$-2.726517$

0.725220

H 2.546410

1.756183

$-0.828220$

$\mathrm{H} \quad 1.712470$

2.517813

1.255721

H -1.178392

H $\quad-0.645730$

2.710644

2.545406

0.16

$-1.289487$

\section{6}

Complex 1a (PCM)

U $\quad-0.085028-0.011227 \quad 0.003993$

$\begin{array}{llll}\text { O } & -0.149999 & -0.029241 & -1.812491\end{array}$

O $-0.141152-0.044097 \quad 1.821079$

$\begin{array}{llll}\mathrm{O} & 0.366234 & 2.460354 & 0.027169\end{array}$

$\begin{array}{lllll}\text { O } & 0.532452 & -2.440594 & -0.078759\end{array}$

$\begin{array}{llll}\mathrm{O} & -2.215733 & 1.411072 & -0.014613\end{array}$

$\begin{array}{llll}\mathrm{O} & -2.233515 & -1.350280 & 0.068279\end{array}$

$\begin{array}{llll}\mathrm{Cl} & 2.644614 & 0.080448 & 0.002282\end{array}$

$\begin{array}{llll}\mathrm{H} & 0.919245 & 2.851625 & -0.698298\end{array}$

H $\quad 0.707485 \quad 2.871024 \quad 0.864005$

H $\quad 1.067394 \quad-2.752438 \quad-0.854526$

H $\quad 0.976466 \quad-2.836664 \quad 0.715913$

$\begin{array}{llll}\mathrm{H} & -3.029880 & 0.989294 & -0.385051\end{array}$

$\begin{array}{llll}\mathrm{H} & -2.158449 & 2.302407 & -0.439954\end{array}$

H $\quad-2.379318 \quad-2.049778 \quad-0.619518$

$\mathrm{H} \quad-2.505110 \quad-1.767876 \quad 0.926001$

13

Complex 1b

$\begin{array}{rrrr}\mathrm{O} & 0.393897 & -0.122122 & -1.560839 \\ \mathrm{U} & 0.450963 & -0.385299 & 0.228695 \\ \mathrm{O} & 0.840560 & -0.845178 & 1.935033 \\ \mathrm{Cl} & -1.737683 & 0.910812 & 0.777941 \\ \mathrm{O} & 2.573897 & -1.643672 & -0.302322 \\ \mathrm{O} & -0.987618 & -2.431911 & -0.009761 \\ \mathrm{O} & 1.653218 & 1.800983 & 0.526221 \\ \mathrm{H} & 3.157255 & -2.090168 & 0.341979 \\ \mathrm{H} & 2.963348 & -1.771029 & -1.189384 \\ \mathrm{H} & -1.393778 & -2.885888 & 0.755604 \\ \mathrm{H} & -1.588787 & -2.571327 & -0.768707 \\ \mathrm{H} & 1.788141 & 2.215980 & 1.401697 \\ \mathrm{H} & 1.592374 & 2.530698 & -0.122395\end{array}$

14

Complex cis-2a

$\begin{array}{rrrc}\mathrm{U} & -0.029586 & -0.102764 & -0.180409 \\ \mathrm{O} & -0.024102 & -0.081234 & 1.641345 \\ \mathrm{O} & -0.345108 & 0.119035 & -1.955881 \\ \mathrm{Cl} & 2.574797 & 0.634521 & -0.464271 \\ \mathrm{O} & -2.296937 & -1.400594 & 0.087000 \\ \mathrm{Cl} & 0.454347 & -2.751788 & -0.386679 \\ \mathrm{O} & -2.140637 & 1.527493 & -0.125145 \\ \mathrm{O} & 0.351174 & 2.584827 & 0.276181 \\ \mathrm{H} & -1.921618 & -2.295690 & -0.088909 \\ \mathrm{H} & -2.510866 & -1.402345 & 1.041551 \\ \mathrm{H} & -2.564989 & 1.432057 & -1.000469 \\ \mathrm{H} & -1.646834 & 2.375806 & -0.163984 \\ \mathrm{H} & 1.248733 & 2.541860 & -0.137883 \\ \mathrm{H} & 0.540016 & 2.575982 & 1.236891\end{array}$


11

Complex cis-2b

O 0.383786

U $\quad 0.169361$

0.092400

1.807677

Cl -1.810702

O 0.432329

0.019669

1.805704

0.024884

2.205997

O 1.535928

$\mathrm{Cl}-1.161754$

$-2.291674$

O 2.142578

$-1.639576$

$\mathrm{H} \quad 2.075190 \quad-2.245293$

H 2.056373

H 1.294165

H 1.269348

$-2.212407$

2.726753

2.759010
0.001286

$-0.058487$

$-1.800146$

$-0.022195$

0.026479

0.059876

$-0.705600$

0.848427

$-0.814479$

0.739697
14

Complex trans-2a

$\begin{array}{rrr}\mathrm{O} & 0.116043 & -0.078818 \\ \mathrm{U} & -0.012252 & -0.095734 \\ \mathrm{O} & 0.749616 & 2.368342 \\ \mathrm{O} & -0.215096 & -0.287916 \\ \mathrm{Cl} & 2.718928 & -0.253273 \\ \mathrm{O} & -2.122926 & -1.663135 \\ \mathrm{O} & 0.717097 & -2.616137 \\ \mathrm{Cl} & -2.294734 & 1.445760 \\ \mathrm{H} & -2.320426 & -1.983763 \\ \mathrm{H} & -2.784031 & -0.950617 \\ \mathrm{H} & 1.678604 & -2.481618 \\ \mathrm{H} & 0.657730 & -2.855451 \\ \mathrm{H} & 0.066753 & 2.918884 \\ \mathrm{H} & 1.593893 & 2.518557\end{array}$

1.684610

$-0.127925$

$-0.220783$

$-1.921170$

$-0.310588$

0.034965

0.091550

0.080795

$-0.867694$

0.198472

$-0.075239$

1.037940

0.214057

0.248212

12

Complex cis-3a
U 0.003753
0.04369
$-0.000361$
O 0.183319
$-0.186959$
1.800694
O $\quad-0.292258$
$-0.083527$
$-1.796313$
Cl 2.756981
$-0.635196$
$-0.344598$
O $\quad-2.040618$
$-1.782657$
0.106820
O $\quad 0.581627$
$-2.638013$
$-0.043463$
$\mathrm{Cl}-2.619076$
1.123569
0.337923
$\mathrm{Cl} \quad 0.837640$
2.606405
$-0.036866$
$\mathrm{H}-2.033963$
$-1.982257$
$-0.850048$
H $\quad-2.647769$
$-0.995629$
0.193613
H 1.534723
$-2.361113$
$-0.146083$
H 0.467648
$-2.770696$
0.918248

9

Complex 3b

14

O 0.044403

$\begin{array}{ll}0.267579 & 1.681973\end{array}$

U $\quad-0.200160$

0.066812

$-0.116510$

Cl -2.329283

1.750640

$-0.003213$

O -0.271198

0.044738

$-1.932686$

O 1.500129

2.111302

$-0.118164$

Cl -1.505618

$-2.287077$

0.138378

$\mathrm{Cl} 2.501482$

$-0.780872$

$-0.293138$

H 2.221355

1.438482

$-0.262364$

H 1.508230

2.245078

0.851435

$\begin{array}{llll}\mathrm{Cl} & 2.650313 & -0.773184 & 0.052044\end{array}$

$\begin{array}{llll}\mathrm{H} & 1.120183 & 2.868772 & -0.655639\end{array}$

$\mathrm{H} \quad 2.410853 \quad 2.052735 \quad-0.291120$

$\begin{array}{llll}\mathrm{H} & -2.132655 & 2.255737 & -0.593771\end{array}$

$\begin{array}{llll}\mathrm{H} & -1.597763 & 2.600890 & 0.842528\end{array}$

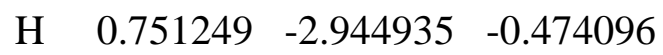

$\begin{array}{llll}\mathrm{H} & -0.818759 & -2.918291 & -0.459764\end{array}$ 


\begin{tabular}{lrrr}
\multicolumn{4}{l}{7} \\
\multicolumn{4}{c}{ Complex 4 } \\
$\mathrm{O}$ & -0.038679 & 0.025566 & 1.825302 \\
$\mathrm{U}$ & -0.013767 & -0.009834 & -0.000503 \\
$\mathrm{Cl}$ & -2.293479 & 1.645797 & -0.063705 \\
$\mathrm{O}$ & 0.011165 & -0.045218 & -1.826308 \\
$\mathrm{Cl}$ & 1.642658 & 2.270082 & -0.022118 \\
$\mathrm{Cl}$ & -1.670227 & -2.289715 & 0.021093 \\
$\mathrm{Cl}$ & 2.265867 & -1.665576 & 0.062723
\end{tabular}

10

Complex $\left[\mathrm{UO}_{2}\left(\mathrm{H}_{2} \mathrm{O}\right) \mathrm{Cl}_{4}\right]^{2-}(\mathrm{PCM})$

$\begin{array}{llll}\text { U } & -0.001336 & -0.022553 & 0.011488\end{array}$

$\begin{array}{llll}\text { O } & -0.000436 & -0.088160 & -1.814605\end{array}$

$\begin{array}{llll}\text { O } & -0.001359 & -0.021958 & 1.839319\end{array}$

$\begin{array}{llll}\text { O } & 0.006294 & -2.654449 & 0.111239\end{array}$

Cl $\quad-2.622489-1.275877 \quad-0.015052$

$\begin{array}{llll}\mathrm{Cl} & 2.629358 & -1.266125 & -0.026261\end{array}$

$\begin{array}{llll}\mathrm{Cl} & 1.790326 & 2.161848 & -0.013585\end{array}$

$\begin{array}{lllll}\mathrm{Cl} & -1.794464 & 2.158203 & -0.029759\end{array}$

$\mathrm{H} \quad-0.770206 \quad-3.021843 \quad-0.373041$

H $\quad 0.810725 \quad-3.013587 \quad-0.332317$
18

Complex 5 (PCM)

$\begin{array}{llll}\text { U } & -0.011804 & -0.003019 & 0.001785\end{array}$

$\begin{array}{llll}\mathrm{O} & -0.083259 & -0.008393 & 1.811741\end{array}$

$\begin{array}{llll}\text { O } & 2.495295 & 0.016673 & -0.155251\end{array}$

$\begin{array}{llll}\text { O } & -0.090911 & -0.017684 & -1.807463\end{array}$

$\begin{array}{llll}\text { O } & 0.930148 & -2.291037 & 0.118608\end{array}$

$\begin{array}{llll}\text { O } & 0.925440 & 2.290425 & 0.189069\end{array}$

$\begin{array}{lllll}\mathrm{O} & -1.983765 & -1.489822 & -0.003782\end{array}$

$\begin{array}{llll}\mathrm{O} & -1.953690 & 1.522982 & -0.129467\end{array}$

$\mathrm{H} \quad 2.975250 \quad-0.764257 \quad-0.532819$

$\mathrm{H} \quad 2.962532 \quad 0.819244 \quad-0.503473$

H $\quad 0.912149 \quad-2.826542 \quad 0.955308$

H $\quad 0.832228-2.942763 \quad-0.625347$

$\mathrm{H} \quad 0.969120 \quad 2.758619 \quad 1.064460$

$\mathrm{H} \quad 0.719010 \quad 2.992555 \quad-0.483507$

H $\quad-2.513158 \quad-1.692682 \quad 0.811551$

$\mathrm{H} \quad-2.614235 \quad-1.557072 \quad-0.768225$

$\mathrm{H} \quad-2.525043 \quad 1.733809 \quad 0.655260$

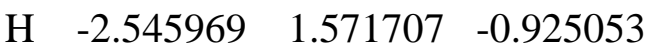

18

Complex 5

$\begin{array}{lrrr}\mathrm{U} & -0.013701 & -0.009793 & 0.000028 \\ \mathrm{O} & -0.037842 & 0.023649 & 1.793637 \\ \mathrm{O} & 0.010690 & -0.042904 & -1.793580 \\ \mathrm{O} & 2.524553 & -0.010114 & 0.033090 \\ \mathrm{O} & -2.067486 & -1.502119 & 0.000090 \\ \mathrm{O} & 0.770076 & -2.423885 & 0.056945 \\ \mathrm{O} & -2.067227 & 1.481370 & -0.056695 \\ \mathrm{O} & 0.770294 & 2.404423 & -0.033267 \\ \mathrm{H} & -2.358515 & -2.094187 & -0.724127 \\ \mathrm{H} & -2.720696 & -1.596011 & 0.724137 \\ \mathrm{H} & 1.237152 & -2.903078 & -0.658562 \\ \mathrm{H} & 0.663315 & -3.054478 & 0.799040 \\ \mathrm{H} & -2.699695 & 1.574779 & -0.798989 \\ \mathrm{H} & -2.378825 & 2.073588 & 0.658795 \\ \mathrm{H} & 3.117930 & 0.265382 & -0.696122 \\ \mathrm{H} & 3.098201 & -0.285712 & 0.777885 \\ \mathrm{H} & 0.685558 & 3.035170 & -0.778050 \\ \mathrm{H} & 1.216077 & 2.883318 & 0.695897\end{array}$

